\title{
Information and Cosmological Objects
}

\author{
Igor Gurevich \\ The Institute of Informatics Problems of the Russian Academy of Sciences, Hetnet Consulting Corp., Moscow, Russia \\ *Corresponding Author: iggurevich@gmail.com
}

Copyright $@ 2013$ Horizon Research Publishing All rights reserved.

\begin{abstract}
Estimation of the volume of information in cosmological objects, including stars of the Sun type, neutron stars, white dwarfs, black holes is necessary for generation of restrictions for their formation, development and interconversion. Information is an integral part of the Universe. The basic law of Zeilinger's quantum mechanics postulates that the elementary physical system (in particular, fundamental particles: quark, electron, photon) bears one bit of information. By its physical essence information is heterogeneity of matter and energy. Therefore information is inseparably connected with matter and energy. An information approach along with a physical one allows to obtain new, sometimes more general data in relation to data obtained on the ground of physical rules only. The author's works, testify about the practicality of information laws usage simultaneously with physical rules for cognition of the Universe. The results presented in this paper show the effectiveness of informational approach for studying the cosmological objects. In future the proposed models and estimations should undoubtedly be specified and presented in more detailed way. One should point out that informational approach allows formulating restrictions on the valuations of physical systems characteristics and physical processes while the physical methods and models can describe not only the restrictions but also concrete physical "mechanisms" of restriction formation, concrete valuations of physical systems characteristics.
\end{abstract}

Keywords Cosmological Objects, Sun, Neutron Stars, White Dwarfs, Black Holes, Information, Heterogeneity, Objective Reality, Restrictions, The Universe

\section{Introduction}

Estimation of the volume of information in cosmological objects, including stars of the Sun type, neutron stars, white dwarfs, black holes is necessary for generation of restrictions for their formation, development and interconversion.

Information is an integral part of the Universe. The basic principle of Zeilinger's quantum mechanics [1] postulates that the elementary physical system (in particular, fundamental particles: quark, electron, photon) bears one bit of information. By its physical essence information is heterogeneity of matter and energy. Therefore information is inseparably connected with matter and energy. The universal measure of physical heterogeneity of information is the Shannon information entropy [2, 3]. It is important to note that the Neumann entropy cannot be applied as the universal measure of heterogeneity because it is equal to zero for structured pure state. An information approach along with a physical one allows to obtain new, sometimes more general data in relation to data obtained on the ground of physical rules only. The author's works, for instance the $[4,5]$ testify about the practicality of information laws usage simultaneously with physical rules for cognition of the Universe. The estimates cited below are based on the foundational principle of Zeilinger quantum mechanics i.e. "an elementary system carries one bit of information" and prove it. The elementary systems are fundamental particles (quarks, leptons, photons). Let us evaluate the volume of information in the system of $n$ q-bits. At first we consider the systems with equiprobable basic states.

\section{Non Interacting Q-bits in the System}

Suppose that the system contains $n$ non-interacting q-bits. Let the q-bit be described by the wave function $\psi=\frac{1}{\sqrt{2}}(|0\rangle+|1\rangle)$ , where $|0\rangle,|1\rangle$ - are the basic states of the q-bit [6]. While measuring the q-bit we'll obtain the basic states $|0\rangle,|1\rangle$ with equal probabilities $1 / 2$. Uncertainty (information) of the q-bit in the state $\psi$ is equal to 1 bit:

$$
N_{1}=I_{1}=-\left(\frac{1}{2} \log _{2} \frac{1}{2}+\frac{1}{2} \log _{2} \frac{1}{2}\right)=1 .
$$

Hence, the volume of information in the system containing $n$ non-interacting q-bits with equiprobable basic states is proportional to the amount of q-bits and is equal to $\mathrm{n}$ bit.

This estimate determines the minimum volume of information in the system consisting of $n$ q-bits with equiprobable basic states. It also explains the linear dependence of the volume of information on the mass or 
number of particles (elementary systems) in the usual substance (fundamental particles - quarks, leptons, photons).

\section{The System Having N Basic States}

Let us consider the case when the objects in the system are specified by the wave functions $\psi=\frac{1}{\sqrt{n}}\left(\sum_{i=1}^{n}\left|e_{i}\right\rangle\right)$. Uncertainty (information) of the object $i$ is equal to $N_{i}=-\left(\frac{1}{n} \log \frac{1}{n}+\ldots+\frac{1}{n} \log \frac{1}{n}\right)=\log _{2} n$. If the system has $n$ basic states, then the system uncertainty (information) content is equal to

$N=\sum_{i=1}^{n} N_{i}=-n\left(\frac{1}{n} \log \frac{1}{n}+\ldots+\frac{1}{n} \log \frac{1}{n}\right)=n \log _{2} n$.

This dependence characterizes the neutron stars and white dwarfs [7]. The neutron stars and white dwarfs appear to be degenerate fermionic systems that fill in a zone of $\mathrm{n}$ states $\left|e_{i}\right\rangle$.

\section{Q-bits Pairwise Interaction in the System}

Suppose that a system contains $n$ pairwise interacting q-bits with equiprobable basic states. The system consisting of $n$ interacting particles (q-bits) can be described with the following wave function: $\psi_{n}^{+}=\frac{1}{\sqrt{2}}\left(\left|0_{1}\right\rangle\left|0_{2}\right\rangle \ldots\left|0_{n}\right\rangle+\left|1_{1}\right\rangle\left|1_{2}\right\rangle \ldots\left|1_{n}\right\rangle\right)$. Each q-bit $i$ possesses the wave function $\psi_{i}=\frac{1}{\sqrt{2}}\left(\left|0_{i}\right\rangle+\left|1_{i}\right\rangle\right)$ $\left(\left|0_{i}\right\rangle,\left|1_{i}\right\rangle\right.$ - basic states of the $i$-th q-bit). Information on relationships between each pair of interacting q-bits $i, j$ is equal to one unit (one bit) [8]. Let us demonstrate this. Under the linked (intricate) status of q-bits $i, j$ the status of q-bit $j$ is absolutely certain if the status of q-bit $i$ is known, and vice versa the status of q-bit $i$ is absolutely certain if the status of q-bit $j$ is known.

For the state $\psi=\frac{1}{\sqrt{2}}\left(\left|0_{i}\right\rangle\left|0_{j}\right\rangle+\left|1_{i}\right\rangle\left|1_{j}\right\rangle\right) \quad$ the probabilities of basic states realization are equal to $P\left(\left|0_{i}\right\rangle\right)=P\left(\left|1_{i}\right\rangle=\frac{1}{2} \quad, \quad P\left(\left|0_{j}\right\rangle\right)\right)=P\left(\left|1_{j}\right\rangle\right)=\frac{1}{2} \quad ; \quad$ the probabilities of pairwise states realization are equal to $P\left(\left|0_{i}\right\rangle\left|0_{j}\right\rangle\right)=P\left(\left|1_{i}\right\rangle\left|1_{j}\right\rangle\right)=\frac{1}{2}$. Combined probabilities are defined with matrix $\mathrm{P}=\left(\begin{array}{cc}\frac{1}{2} & 0 \\ 0 & \frac{1}{2}\end{array}\right)$. Indefiniteness (information entropy) of q-bits $i, j$ is equal to $N_{i}=N_{j}=-\left(\frac{1}{2} \log \frac{1}{2}+\frac{1}{2} \log \frac{1}{2}\right)=1 \quad$ bit. Indefiniteness (information entropy) of combined distribution of the states of q-bit $i$ and q-bit $j$ is equal to $N_{i j}=-\left(\frac{1}{2} \log \frac{1}{2}+\frac{1}{2} \log \frac{1}{2}\right)=1$ (bit). Information on the links of q-bits $i$ and $j$ is equal to $I_{i j}=N_{i}+N_{j}-N_{i j}=1+1-1=1$ bit.

The volume of information in the link of a system of $n$ pairwise interacting q-bits with equiprobable basic states described with the wave function

$$
\begin{aligned}
& \psi_{n}=\frac{1}{\sqrt{2}}\left(\left|0_{1}\right\rangle\left|0_{2}\right\rangle \ldots\left|0_{n}\right\rangle+\left|1_{1}\right\rangle\left|1_{2}\right\rangle \ldots\left|1_{n}\right\rangle\right) \\
& I_{n}=\frac{n \cdot(n-1)}{2} \text { bit. }
\end{aligned}
$$

is equal to $\begin{array}{lll}n & \text { bit. }\end{array}$

The volume of information in the system of $n$ pairwise interacting q-bits is formed from $n$ bits of information available in the q-bits and $\frac{n \cdot(n-1)}{2}$ bit of information on the link between the q-bits. The total volume of information in the system containing $n$ pairwise interacting q-bits with equiprobable basic states is equal to

$$
I_{n}=n+\frac{n \cdot(n-1)}{2}=\frac{n \cdot(n+1)}{2} \text { bit. }
$$

This estimate determines the maximum volume of information in the system consisting of $n$ q-bits with equiprobable basic states. In the system containing $n$ pairwise interacting q-bits with equiprobable basic states the volume of information is proportional to squared number of q-bits and is equal to $I_{n}=\frac{n \cdot(n+1)}{2}$ bit.

The volume of information in a system of $n$ pairwise interacting q-bits with equiprobable basic states is $n$ bit more than in a system consisting of $n-1$ q-bit,

$$
I_{n}-I_{n-1}=\frac{n \cdot(n+1)}{2}-\frac{(n-1) \cdot n}{2}=n
$$

Under $n>>1$ the volume of information in a system of $n$ pairwise interacting q-bits is equal to one half of squared number of interacting q-bits $I_{n} \approx \frac{n^{2}}{2}$. This explains 
quadratic dependence of the the volume of information upon the black holes mass.

\section{Local Q-bit Interaction in the System}

Let us consider a case when in the system of ${ }^{n}$ q-bits there stand out $\frac{n}{k}$ groups of $k$ q-bits, each of $k$ q-bits interacting only with the q-bits of each group (suppose that $n$ is divided by $k$ ). Then the volume of information $I_{k}$ in the group of $k$ pairwise interacting q-bits with equiprobable basic states is equal to $\frac{k \cdot(k+1)}{2}$ bit. Hence the system under consideration consisting of $n$ q-bits contains $I_{n / k}=\frac{n}{k} \frac{k \cdot(k+1)}{2}=\frac{n \cdot(k+1)}{2}$

bit. This explains a linear dependence of the volume of information on the mass in the systems composed of compound particles of usual substance (for instance, in the systems composed of elementary particles - mesons, baryons, atoms, molecules, gas, ... ).

At $k=1$ the system contains the minimum information content: $I_{n / 1}=n \frac{1 \cdot(1+1)}{2}=n$. At $k=n$ the system contains the maximum volume of information:

$$
I_{n / n}=\frac{n}{n} \frac{n \cdot(n+1)}{2}=\frac{n \cdot(n+1)}{2} .
$$

\section{Restrictions on the Volume of Informationin the System with Equiprobable and Non-equiprobable Basic States}

In the general case, the volume of information ${ }^{I} n$ in the system of $n$ q-bits with equiprobable basic states is no less than $n$ bit and no more than $\frac{n \cdot(n+1)}{2}$ bit:

$$
n \leq I_{n} \leq \frac{n \cdot(n+1)}{2} .
$$

If we consider the systems consisting of $n$ q-bits with arbitrary probabilities realization of basic states $|0\rangle,|1\rangle$, then a q-bit is described with the wave function $\psi=a|0\rangle+b|1\rangle$. While making the q-bit measurements we obtain the basic states $|0\rangle,|1\rangle$ with probabilities $|a|^{2},|b|^{2}$. Uncertainty (information) of the q-bits in the state $\psi$ is equal to $N_{1}=I_{1}=-\left(|a|^{2} \log _{2}|a|^{2}+|b|^{2} \log _{2}|b|^{2}\right)$.

In the general case, the volume of information $I_{n}$ in the system of $n$ q-bits is larger or equal to zero bits and is not larger than $\frac{n \cdot(n+1)}{2}$ bit: $0 \leq I_{n} \leq \frac{n \cdot(n+1)}{2}$.

\section{The Volume of Information in the Black Hole. Information Model of the Black Hole}

The information volume of a black hole of mass $M_{B h}$ is equal to $I_{B h}=\frac{2 \pi \cdot G}{\hbar \cdot c} M_{B h}^{2}$ Nat, or $I_{B h}=\frac{2 \pi \cdot G}{\hbar \cdot c \cdot \ln 2} M_{B h}^{2}$ bit [9]. The information volume contained in the black hole is proportional to its squared mass. How to explain it? Let us assume that a black hole contains $n$ pairwise interacting particles (q-bits). Then the quadratic dependence of the volume of information in the black hole on its mass can be explained by the fact that each interaction forms 1 bit of information. The black hole is described with the wave

function

$$
\psi_{n}=\frac{1}{\sqrt{2}}\left(\left|0_{1}\right\rangle\left|0_{2}\right\rangle \ldots\left|0_{n}\right\rangle+\left|1_{1}\right\rangle\left|1_{2}\right\rangle \ldots\left|1_{n}\right\rangle\right)
$$

mentioned earlier the volume of information in the system described with the present given function is equal to $I_{n}=\frac{n \cdot(n+1)}{2}$

bit. At $n>>1$ the volume of information in the black hole is proportional to the black hole squared

$$
I_{n} \approx \frac{n^{2}}{2} \text {. }
$$
contains the information, wh hole consisting of $n-1$ particles:

$$
I_{n}-I_{n-1}=\frac{n(n+1)}{2}-\frac{(n-1) \cdot n}{2}=n .
$$

Let us compare the estimates of squared mass of a black hole: $M_{B h}^{2}=\frac{\hbar \cdot c \cdot \ln 2}{2 \pi \cdot G} \cdot I_{B h} \quad$ (from cosmological formula [9]) and $M_{B h}^{2}=n^{2} \cdot m_{0}^{2}$ (squared mass $n$ of the particles composing the black hole). We have $M_{B h}^{2}=n^{2} \cdot m_{0}^{2}=\frac{\hbar \cdot c \cdot \ln 2}{2 \pi \cdot G} \cdot I_{B h}$. As far as $I_{B h}=\frac{n^{2}}{2}$, then $n^{2} \cdot m_{0}^{2}=\frac{\hbar \cdot c \cdot \ln 2}{2 \pi \cdot G} \cdot \frac{n^{2}}{2} \quad, \quad$ or $\quad m_{0}^{2}=\frac{1}{4 \pi} \cdot \frac{\hbar \cdot c \cdot \ln 2}{G}$ Eventually we obtain the estimate of mass of particles being part of the black holes: $m_{0}=\frac{\sqrt{\ln 2}}{2 \sqrt{\pi}} \sqrt{\frac{\hbar \cdot c}{G}}$, that agree by its 
magnitude with Planck mass $m_{P l}=\sqrt{\frac{\hbar \cdot c}{G}} \approx 2,17 \cdot 10^{-5} \mathrm{~g}:$
\[ m_{0}=\frac{\sqrt{\ln 2}}{2 \sqrt{\pi}} m_{P l} \approx 0,23 \sqrt{\frac{\hbar \cdot c}{G}}=5,09 \cdot 10^{-6} \mathrm{~g} . \]

Therefore, a black hole is the aggregate of particles (let us call them black particles) each having a mass equal to 0.23 th of Planck mass) and interacting with all other black particles that form a black hole $[5,10]$.

\section{Emission and Absorption of Usual Substance by a Black Hole}

Suppose that at the initial instant of time a black hole consisting of $n$ black particles has the mass of $M_{n}=n \cdot m_{0} \quad$ and contains $\quad I_{n}=\frac{n \cdot(n+1)}{2}$ bit of information. The black hole mass changes (increases or decreases) by black particles (quanta) $\Delta M=m_{0}$. As far as each black hole has the sub-Planck mass $m_{0}$, then the change in energy of a black hole in such a case is equal to $\Delta E=m_{0} c^{2}$. In the event of the loss of emission of one black particle the mass of a black hole becomes equal to $M_{n}=(n-1) \cdot m_{0}$. In this case the volume of information remaining in the black hole is $I_{n-1}=\frac{(n-1) \cdot n}{2}$ bit. Change (loss) of information in the black hole comes to $n$ bit.

For further estimates we implement the law of conservation of uncertainty (information) $[4,5]$ and energy conservation principle. According to the law of conservation of uncertainty (information) a change in the system "a black hole with the mass $M_{n}=n \cdot m_{0}$ - external environment" on emission of one black particle must be balanced by the occurrence of $n$ particles containing 1 bit each: $I_{n}-I_{n-1}=n$ bit. It can believed that $n$ photons of frequency $v$ and energy $h v$ emerged.

In virtue of energy conservation principle $n \cdot h v=m_{0} c^{2}$

$$
\text { and } v=\frac{m_{0} c^{2}}{n \cdot h} \text {. Because } \quad m_{0}=\frac{\sqrt{\ln 2}}{2 \sqrt{\pi}} \sqrt{\frac{\hbar \cdot c}{G}},
$$
frequency of emitted photon must be equal to

$$
v=\frac{1}{n} \cdot \frac{\sqrt{\ln 2}}{4 \pi \sqrt{\pi}} \sqrt{\frac{c^{5}}{\hbar \cdot G}}=\frac{0,69 \cdot 10^{42}}{n}
$$

1/c. Because
$t_{P l}=\sqrt{\frac{\hbar \cdot G}{c^{5}}} \approx 5,41 \cdot 10^{-44} \mathrm{c}$, then $\quad v=\frac{1}{n} \cdot \frac{\sqrt{\ln 2}}{4 \pi \sqrt{\pi} \cdot t_{P l}}$.

Let us

denote

$v_{0}=\frac{\sqrt{\ln 2}}{4 \pi \sqrt{\pi}} \frac{1}{t_{P l}}=\frac{\sqrt{\ln 2}}{4 \pi \sqrt{\pi}} \sqrt{\frac{c^{5}}{\hbar \cdot G}} \approx 0,68 \cdot 10^{42}$

1/c. Then $v=\frac{v_{0}}{n}$.

In the case of a black hole containing one black particle the radiation frequency is maximal and in inverse proportion to Planck time unit. Similar dependences are true for absorption of photons by black holes.

Note 1. In the general case, there must appear $k$ particles with aggregated information equal to $n$ bit and aggregated energy $m_{0} c^{2}$.

Note 2. In the general case, there must be absorbed $n$ photons having the frequencies $v_{i}$ and the aggregated energy $h \sum_{i=1}^{n} v_{i}=m_{0} c^{2}$ equal to $n$ bit.

Note 3. In the general case, there must be absorbed $k$ particles with aggregated information equal to $n$ bit and aggregated energy $m_{0} c^{2}$.

Identical dependencies are true for cases when photons are absorbed by black holes. In virtue of the law of conservation of uncertainty (information), the changes in the system "a black hole with the mass $M_{n-1}=(n-1) \cdot m_{0}$ - external environment", when the mass of a black hole is increased by the mass of one black particle, must be determined by absorption of $n$ particles containing 1 bit each: $I_{n}-I_{n-1}=n$ bit. Suppose that there had been absorbed $n$ photons of frequency $v$ and energy $h v$. By virtue of energy conservation principle $n \cdot h v=m_{0} c^{2}$. The frequency of each absorbed photon must be equal to $v=\frac{1}{n} \cdot \frac{\sqrt{\ln 2}}{4 \pi \sqrt{\pi}} \sqrt{\frac{c^{5}}{\hbar \cdot G}}=\frac{0,69 \cdot 10^{42}}{n}$ of a black hole consisting one black particle the absorption frequency is maximal and inversely proportional to Planck time unit.

Having the estimates of black holes distribution by mass one can calculate the intensity of aggregated distribution of black holes radiation by frequencies and compare them with the experiment results. From the obtained radiation frequency expression one can draw the estimate of black hole radiation temperature.

Let us calculate the radiation temperature 


$$
T=\frac{h v}{2.7 k}=\frac{h}{2,7 k} \frac{1}{n} \cdot \frac{\ln 2}{8 \pi^{2}} \frac{c^{3}}{\mathrm{M} \cdot \mathrm{G}}=\frac{\ln 2 \cdot \hbar \cdot c^{3}}{4 \pi \cdot 2,7 \cdot k \cdot \mathrm{M} \cdot \mathrm{G}}
$$

\section{Formation and Development (Changing) of Black Holes}

Let us assume that a black hole of mass $M_{n}=n \cdot m_{0}$ is formed from the usual substance (radiation). As mentioned earlier, such a black hole contains $I=\frac{n(n+1)}{2}$ bit of information. Therefore, for its formation are needed $\underline{n(n+1)}$

2 particles of usual substance (radiation quanta), each of them containing 1 bit. For formation of a black hole

of the mass equal to $M \mathrm{~kg}$ it is necessary to form $n=\frac{M}{m_{0}}$ sub Planck particles and use $\frac{n(n+1)}{2}=\frac{\frac{M}{m_{0}}\left(\frac{M}{m_{0}}+1\right)}{2} \approx \frac{M^{2}}{2 m_{0}^{2}}$ for formation of a black hole with the mass of the Sun equal to $\approx 2 \cdot 10^{30} \mathrm{~kg}, \quad$ it is necessary to form $n=\frac{M_{\text {sun }}}{m_{0}}=\frac{2 \cdot 10^{33}}{5 \cdot 10^{-6}}=4 \cdot 10^{38}$ and use $\frac{n(n+1)}{2} \approx 8 \cdot 10^{76}$ radiation quanta.

It is known that during supernova explosion the radiation energy is on the order of $10^{50} \mathrm{erg}$, the maximum $10^{54} \mathrm{erg}$ [11]. Whereas the average radiation frequency is $10^{18} \mathrm{~Hz}$, then the supernova explosion generates $N=\frac{10^{50}\left(10^{54}\right)}{10^{-27} 10^{18}}=10^{59}\left(10^{63}\right)$

radiation quanta. Assuming that $\approx 100 \%$ radiation quanta is used for black hole formation, then the mass of a black hole would be equal to about $M \approx \sqrt{2 N} m_{0} \approx 2,3 \cdot 10^{24} \mathrm{~g}$.

The table 1 presents information characteristics of black holes of different masses.

Table 1. Information characteristics of black holes of different masse

\begin{tabular}{|c|c|}
\hline $\begin{array}{l}\text { The number of absorbed radiation quanta } \\
\text { (the volume of absorbed information) }\end{array}$ & $\begin{array}{l}\text { The number of sub Planck particles in the } \\
\text { newly formed black hole }\end{array}$ \\
\hline 1 & 2 \\
\hline 1 & 1 \\
\hline $1 \mathrm{E}+10$ & 141421 \\
\hline $1 \mathrm{E}+20$ & 14142135623 \\
\hline $1 \mathrm{E}+30$ & $1,41421 \mathrm{E}+15$ \\
\hline $3,76 \mathrm{E}+38$ & $1,78326 \mathrm{E}+19$ \\
\hline $1 E+40$ & $1,41421 \mathrm{E}+20$ \\
\hline $1 \mathrm{E}+50$ & $1,41421 \mathrm{E}+25$ \\
\hline $1 E+59$ & $1,47214 \mathrm{E}+29$ \\
\hline $1 \mathrm{E}+60$ & $1,41421 \mathrm{E}+30$ \\
\hline $1,26 \mathrm{E}+62$ & $1,58745 \mathrm{E}+31$ \\
\hline $1 E+63$ & $4,47214 \mathrm{E}+31$ \\
\hline $1 \mathrm{E}+70$ & $1,41421 \mathrm{E}+35$ \\
\hline $7,72 \mathrm{E}+76$ & $3,92927 \mathrm{E}+38$ \\
\hline $1 \mathrm{E}+80$ & $1,41421 \mathrm{E}+40$ \\
\hline $1 \mathrm{E}+90$ & $1,41421 \mathrm{E}+45$ \\
\hline $7,72 \mathrm{E}+94$ & $3,92927 \mathrm{E}+47$ \\
\hline $1 \mathrm{E}+100$ & $1,41421 \mathrm{E}+50$ \\
\hline $7,72 \mathrm{E}+100$ & $3,92927 \mathrm{E}+50$ \\
\hline $1 \mathrm{E}+110$ & $1,41421 \mathrm{E}+55$ \\
\hline $1 \mathrm{E}+120$ & $1,41421 \mathrm{E}+60$ \\
\hline
\end{tabular}


Table 1. continued

\begin{tabular}{|c|c|}
\hline $\begin{array}{l}\text { The mass of a newly formed black hole } \\
\qquad(\mathrm{kg})\end{array}$ & Note \\
\hline 3 & 4 \\
\hline 5,09E-09 & Characteristics of the minimal black hole \\
\hline \multicolumn{2}{|l|}{0,000719832} \\
\hline \multicolumn{2}{|l|}{71,98347} \\
\hline \multicolumn{2}{|l|}{$7,2 \mathrm{E}+06$} \\
\hline $1,78 \mathrm{E}+11$ & $\begin{array}{l}\text { Characteristics of the optimal black hole in the system } \\
\text { "hydrogen (protons)- black hole) }\end{array}$ \\
\hline \multicolumn{2}{|l|}{$7,2 \mathrm{E}+11$} \\
\hline \multicolumn{2}{|l|}{$7,2 \mathrm{E}+16$} \\
\hline $2,28 \mathrm{E}+21$ & $\begin{array}{c}\text { Characteristics of a black hole generated during the supernova explosion of radiation } \\
\text { rate of } 10^{50} \text { erg. }\end{array}$ \\
\hline \multicolumn{2}{|l|}{$7,2 \mathrm{E}+21$} \\
\hline $9,09 \mathrm{E}+22$ & $\begin{array}{l}\text { Characteristics of the optimal black hole in the system } \\
\text { "radiation - black hole". See }[5,10,12,13]\end{array}$ \\
\hline $2,28 \mathrm{E}+23$ & $\begin{array}{l}\text { Characteristics of a black hole generated during the } \\
\text { supernova explosion of radiation rate of } 10^{54} \text { erg. }\end{array}$ \\
\hline \multicolumn{2}{|l|}{$7,2 \mathrm{E}+26$} \\
\hline $2 \mathrm{E}+30$ & Characteristics of a black hole having the mass of the Sun \\
\hline \multicolumn{2}{|l|}{$7,2 \mathrm{E}+31$} \\
\hline \multicolumn{2}{|l|}{$7,2 \mathrm{E}+36$} \\
\hline $2 \mathrm{E}+36$ & Characteristics of a black hole of $10^{6}$ of the masses of the Sun \\
\hline \multicolumn{2}{|l|}{$7,2 \mathrm{E}+41$} \\
\hline $2 \mathrm{E}+39$ & Characteristics of a black hole of $10^{9}$ of the masses of the Sun \\
\hline \multicolumn{2}{|l|}{$7,2 \mathrm{E}+46$} \\
\hline $7,2 \mathrm{E}+51$ & Characteristics of the maximum black hole \\
\hline
\end{tabular}

From the above chart it follows that:

1. The mass of a black hole formed during the supernova explosion is close to the mass of an optimal black hole in the system "a black hole - radiation". One can expect that during the supernova explosions the black holes be formed having the masses under which the information volume in the adjacent space is close to minimum.

2. For formation of the black holes with the mass equal to million masses of the Sun the volume of information exceeding the volume of information of the Universe (1090 bit) is required $[4,5,9,14]$. Significant volumes of information are needed for formation of the black holes with the same mass as of the Sun's. In such case the volume of information of about 1076 is required. It means that locally (in the zone of black hole formation) there must take place the intensive physical processes of radiation formation. For instance, the supernova explosions and accelerated motion of relativistic particles.

\section{The Volume of information in the Neutron Stars and White Dwarfs. The Information Model of a Neutron Star and White Dwarf}

When evaluating the volume of information in the neutron star we must take into account the volume of information in the structure of the star and in the neutrons. In the neutron fermi-gas during its complete degeneration all the low energy levels are filled in up to the Fermi level while all the subsequent ones remain empty. Temperature rise can change distribution of neutrons in the levels to only a small extent: a small fraction of neutrons sitting in the levels close to the Fermi level pass to the empty levels possessing bigger energy and clear the levels from which the migration took place.

The volume of information in the white dwarfs and neutron stars. When evaluating the volume of information in the neutron star we must take into account the volume of information in the structure of the star and in the neutrons. The volume of information in the structure of degenerate fermi-gas is equal to $\mathrm{N}^{*} \log 2(\mathrm{~N})$ bit. Here $\mathrm{N}-$ is the number of filled in energy levels (number of neutrons). In the neutron star $\mathrm{N}=(\mathrm{M} / \mathrm{m})$, where $\mathrm{M}-$ is the mass of neutron star, $\mathrm{m}-$ is the mass of neutron. The volume of information in one neutron is about 9,422 bit. The volume of information in the neutrons of the star is equal to $9,422 * \mathrm{~N}$. Aggregated volume of information in the neutron star (without considering the information in the crust nickel and iron) is 
approx. $9,422 *(\mathrm{M} / \mathrm{m})+(\mathrm{M} / \mathrm{m}) * \log 2(\mathrm{M} / \mathrm{m})$ bit. The volume of information in the neutron star is proportional to the mass multiplied by the logarithm of dyadic mass. In a similar way one can evaluate the volume of information in the white dwarfs. Recall for the sake of comparison that the volume of information in the usual substance (non-interacting particles, gas) is proportional to mass, the volume of information in the black hole is proportional to squared mass.

The neutron mass is $m=1,67 \cdot 10^{-27} \mathrm{~kg}$. A number of neutrons in the star with the mass of $1,0(1,4)$ of the mass of the Sun is equal to $\approx 1,18 \cdot 10^{57}\left(\approx 1,68 \cdot 10^{57}\right)$. Therefore, the neutron star with the mass of $1,0(1,4)$ of the mass of the Sun contains $\approx 2,32 \cdot 10^{59}\left(\approx 3,2 \cdot 10^{59}\right)$ bit. The neutron star structure contains the amount of information, which is about two orders of magnitude more $\approx 2,25 \cdot 10^{59}$ $\left(\approx 3,16 \cdot 10^{59}\right)$, than in the neutrons $\approx 6,93 \cdot 10^{57}$ $\left(\approx 9,7 \cdot 10^{57}\right)$.

The most commonly encountered are the white dwarfs consisting of carbon and oxygen with helium-hydrogen shell $[7,15]$. Under the masses of white dwarfs 0.6 Msun 1.44Msun, with the radiuses equal to the Earth's the surface temperature can be relatively high (from $100,000 \mathrm{~K}$ to $200,000 \mathrm{~K})$. The main feature of their construction is a nucleus, its gravity equilibrium being supported by degenerate electron gas whose properties do not allow any further modifications of its structure. The degenerate gas pressure puts into equilibrium the gravitation (under prescribed mass) and the loss of heat resulting from non-degenerate component of the substance does not change this pressure and the losses alone are relatively insignificant.

The fate of the supergiant star remnants depends on the mass of remaining nucleus. When hydrostatic equilibrium breaks down there occurs gravitation collapse (lasting for seconds of fractions of seconds) and if Mkern $<1.4$ Msun, then the nucleus will shrink up to the Earth dimensions and a white dwarf is produced. If $1.4 \mathrm{Msun}<\mathrm{Mkern}<3$ Msun, then the pressure of incumbent layers will be so strong that the electrons are "forced into" protons thus generating neutrons and emitting neutrino $p^{+}+e^{-} \rightarrow n+v_{e}$. The so-called a degenerate neutron gas is generated. The pressure of degenerate neutron gas halts the subsequent shrinkage of the star. However, it appears that part of the neutron stars are formed during supernova outbursts and appear to be the remnants of massive stars that had exploded as the Supernova of the second type.

For evaluating the volume of information available in the white dwarf of the mass of the Sun we assume that the white dwarf contains more or less equal number of atoms of carbon and oxygen. Then the total number of atoms in the white dwarf of the mass of the Sun is equal to

$$
n_{C+O}=\frac{M_{C}}{\left(m_{C}+m_{o}\right) / 2} \approx \frac{2 \cdot 10^{30}}{13 \cdot 1,67 \cdot 10^{-27}} \approx 8,55 \cdot 10^{55}
$$

The number of electrons in the white dwarf of the mass of the Sun is equal to $n_{E} \approx 5,99 \cdot 10^{56}$. Thus the structure of the white dwarf of the mass of the Sun contains $\approx 1,13 \cdot 10^{59}$ bit. The carbon and oxygen atoms of the white dwarf of the mass of the Sun contain $\approx 1,11 \cdot 10^{58}$ bit. Therefore, the white dwarf of the mass of the Sun contains only $\approx 1,24 \cdot 10^{59}$ bit.

Emission and absorption of usual substance by the neutron star. The logic of derivation of estimates in the present section is identical to the logic of derivation of estimates for the black hole. Suppose that at the initial instant of time the neutron star consists of $n$ neutrons, possesses the mass of $M_{n}=n \cdot m_{n}$ and contains $I_{n}=n \log _{2} n+9,422 \cdot n$ bit of information. The mass of neutron star changes (increases or decreases) by neutrons (quanta) $\Delta M=m_{n}$. Because each neutron has the mass $m_{n}$, then the neutron star energy change in this case is equal to $\Delta E=m_{n} c^{2}$. At the loss of emission of one neutron the mass of neutron star becomes equal to $M_{n-1}=(n-1) \cdot m_{n}$. In this case the volume of information remaining in the neutron star is equal to $I_{n}=(n-1) \log _{2}(n-1)+9,422 \cdot(n-1) \quad$ bit. Information change (loss) in the neutron star comes to

$$
\begin{array}{r}
\Delta I_{n}=I_{n}-I_{n-1}=n \log _{2} n+9,422 \cdot n-(n-1) \log _{2}(n-1)-9,422 \cdot(n-1)= \\
=\log _{2} n+(n-1)\left(\log _{2} n-\log _{2}(n-1)\right)+9,422= \\
=\log _{2} n+(n-1)\left(\log _{2}(1+1 /(n-1))+9,422 \approx \log _{2} n+9,422\right. \text { bit. }
\end{array}
$$

For further estimates we use the law of conservation of uncertainty (information) and energy conservation principle. According to the law of conservation of uncertainty (information) any change in the system "neutron star of the mass $M_{n}=n \cdot m_{n}$ - external environment" at the emission of one neutron of a particle must be compensated by occurrence of $\log _{2} n+6,8$ particles containing 1 bit each: $I_{n}-I_{n-1}=\log _{2} n+9,422$ bit. Let us consider that there have emerged $\log _{2} n+6,8$ photons of frequency $v$ and energy $h v$. By virtue of the energy conservation principle

$$
\left(\log _{2} n+9,422\right) \cdot h v=m_{n} c^{2} \text { and } v=\frac{m_{n} c^{2}}{\left(\log _{2} n+9,422\right) \cdot h} .
$$

Let us calculate the radiation temperature $T=\frac{h v}{k}=\frac{h}{k} \frac{m_{n} c^{2}}{\left(\log _{2} n+9,422\right) \cdot h}=\frac{m_{n} c^{2}}{\left(\log _{2} n+9,422\right) \cdot k}$.

Estimate of dependence of the temperature of neutron star radiation on its mass (number of neutrons in the star) is presented in the table 2 . 
Table 2. Estimate of dependence of the temperature of neutron star radiation on its mass

\begin{tabular}{|c|c|c|c|}
\hline Neutron star mass $(\mathrm{kg})$ & $1,67 \mathrm{E}-17$ & $1,67 \mathrm{E}-07$ & $1,67 \mathrm{E}+03$ \\
\hline 1 & 2 & 3 & 4 \\
\hline Number of neutrons in the star $n$ & $1 \mathrm{E}+10$ & $1 \mathrm{E}+20$ & $\mathrm{E}+30$ \\
\hline $\begin{array}{c}\text { Number of generated photons } \\
\log _{2} n+9,422\end{array}$ & 40,02 & 73,23 & 106,45 \\
\hline Radiation temperature & $2,55 \mathrm{E}+11$ & $1,44 \mathrm{E}+11$ & $9,98 \mathrm{E}+11$ \\
\hline
\end{tabular}

Table 2. continued

\begin{tabular}{|c|c|c|c|}
\hline Neutron star mass $(\mathrm{kg})$ & $1,67 \mathrm{E}+13$ & $1,67 \mathrm{E}+23$ & $2,00 \mathrm{E}+30$ \\
\hline 5 & 6 & 7 & 8 \\
\hline Number of neutrons in the star $n$ & $10 \mathrm{E}+40$ & $1 \mathrm{E}+50$ & $1,2 \mathrm{E}+57$ \\
\hline $\begin{array}{c}\text { Number of generated photons } \\
\log _{2} n+9,422\end{array}$ & 139,67 & 172,89 & 196,41 \\
\hline Radiation temperature & $7,65 \mathrm{E}+10$ & $6,21 \mathrm{E}+10$ & $5,47 \mathrm{E}+10$ \\
\hline
\end{tabular}

Note that the given estimate does not take into account the availability of other elements in the neutron stars that decrease the radiation temperature.

Note 4. In the general case there must be generated $\log _{2} n+9,422$ photons having the frequencies of $v_{i}$ and aggregated energy $h \sum_{i=1}^{\log _{2} n+9,422} v_{i}=m_{0} c^{2}$.

Similar dependencies are true for the cases when the neutrons are absorbed by neutron stars. The estimate of radiation temperature of the white dwarf of the mass of the Sun was derived by using information method, which is similar to estimations of black hole and neutron star radiation temperature and is equal to about $3 \mathrm{E}+07 \mathrm{~K}$.

According to the law of conservation of uncertainty (information) the change in the system "neutron star of the mass $M_{n-1}=(n-1) \cdot m_{n}$ - external environment" at the black hole mass increase by the mass of one black particle must be conditioned by the absorption of $\log _{2} n+9,422$ particles containing 1 bit each: $I_{n+1}-I_{n}=\log _{2} n+9,422$ bit. Let us assume that there was absorbed $\log _{2} n+9,422$ photons of frequency $v$ and energy $h v$. By virtue of the energy conservation principle $\left(\log _{2} n+6,8\right) \cdot h v=m_{n} c^{2}$. The frequency of each absorbed photon must be equal to $v=\frac{m_{n} c^{2}}{\left(\log _{2} n+9,422\right) \cdot h}$

Similar dependencies are also true for the white dwarfs.

\section{The Information Volume of the Sun. Information Model of a Star of the Sun Type}

The Sun consists for the most part from hydrogen $(\sim 74 \%$ of its mass) and helium ( $25 \%$ of its mass). The number of hydrogen atoms $n_{H}$ in the Sun amounts to $n_{H}=\frac{M_{\text {Sun }}}{m_{H}}=\frac{2 \cdot 0,74 \cdot 10^{30}}{1,66 \cdot 10^{-27}} \approx 0,9 \cdot 10^{57}$

. The hydrogen atom contains 11,422 bit. The volume of information in the system consisting of $n_{B}$ hydrogen atoms is $11,422 n_{H}$ bit. Therefore, hydrogen in the Sun contains $I_{H} \approx 11,422 \cdot 0,9 \cdot 10^{57} \approx 10^{58}$

bit. The helium atom (two protons, two neutrons, two electrones) contains approx. 40 bit of information. The volume of information available in the system consisting of $n_{H e}$ atoms of helium is equal to 40 $n_{H e}$ bit. Thus, helium in the Sun contains $40 n_{\mathrm{He}}=40 \cdot \frac{0,25 \cdot M_{\mathrm{Sun}}}{m_{H}}=\frac{5 \cdot 10^{30}}{1,66 \cdot 10^{-27}} \approx 3 \cdot 10^{57}$ bit. All in all the Sun contains $\approx 1,3 \cdot 10^{58}$ bit of information.

Note 5. Our Galaxy contains over $10^{11}$ stars, the Universe contains $10^{11}$ galaxies. Consequently, our Galaxy and the stars contain about $10^{69}$ bit while the Universe and the stars contain about $10^{80}$ bit of information.

\section{Formation of Black Holes from the Stars}

Table 3 presents the masses of black holes. Estimations were based on the assumption that the black holes had been formed from the neutron star, white dwarf of the Sun type. Suppose that the original neutron star possesses a mass equal to the mass of the Sun ${ }^{M}=2,00 \mathrm{E}+30 \mathrm{~kg}$, consists of $1,20 \mathrm{E}+57$ neutrons and contains the volume of information equal to ${ }_{n}=n\left(\log _{2} n+9,422\right)=2,38 \mathrm{E}+59$ bit. Following the transformation a black hole and usual substance are formed. The black hole mass is equal to ${ }^{M_{B h}}=k m_{0}$ while the volume of information in the newly formed black hole is

$$
I=\frac{k(k+1)}{2}
$$

equal to 2 . The number of usual substance particles formed as a result of the merging is equal to $x$. The volume of information in the usual substance is equal to $I_{U s}=x$. The average mass of usual substance particle is 
equal to $m$.

From the law of conservation of uncertainty (information) it follows:

$$
I_{n}=n\left(\log _{2} n+9,422\right)=\frac{n(n+1)}{2}+x=I_{B h}+I_{U s} .
$$

Suppose that the main energy of the black holes and usual substance is concentrated in the mass. Then from the energy conservation principle it follows:

$$
M_{n}=n m_{n}=k m_{0}+x m=M_{B h}+M_{U s} .
$$

This we have a system consisting of the two equations $n m_{n}=k m_{0}+x m, \quad n\left(\log _{2} n+9,422\right)=\frac{k(k+1)}{2}+x \quad$ in three unknowns $n, x, m . x \geq 0$. Therefore, $n\left(\log _{2} n+9,422\right) \geq \frac{k(k+1)}{2} \approx \frac{k^{2}}{2}, k \leq \sqrt{2 n\left(\log _{2} n+9,422\right)}$ and $M_{B h}=k m_{0} \leq m_{0} \sqrt{2 n\left(\log _{2} n+9,422\right)}$ (restriction on the mass of a black hole formed from the neutron star). At $n$ $=1,20 \mathrm{E}+57 \quad I_{n}=n\left(\log _{2} n+9,422\right) \quad=2,38 \mathrm{E}+59 \quad$ bit $k \leq \sqrt{2 \cdot 2,38 \cdot 10^{59}}=\sqrt{47,6 \cdot 10^{58}} \approx 7 \cdot 10^{29}$. As far as $m_{0}$ $=5,09 \mathrm{E}-09 \mathrm{~kg}$, then $M_{B h} \leq m_{0} 7 \cdot 10^{29} \approx 3,5 \cdot 10^{21} \mathrm{~kg}$.

Let us consider the following example. Suppose that the neutron star consists of $1,20 \mathrm{E}+57$ neutrons, possesses the mass equal to the mass of the Sun $M_{n}=2,00 \mathrm{E}+30 \mathrm{~kg}$ and contains the volume of information equal to $I_{n}=n\left(\log _{2} n+9,422\right)=2,38 \mathrm{E}+59$ bit. The resulting black hole consists of $\mathrm{k}=3 \mathrm{E}+29$ particles (it has the mass of 1,5 $\mathrm{E}+21 \mathrm{~kg}$ and contains $4,5 \mathrm{E}+58 \mathrm{bit}$ ). The resulting number of usual substance particles (radiation quanta) is equal to $1,9 \mathrm{E}+59$. The total mass of usual substance is equal to $(2,00 \mathrm{E}+30-1,5 \mathrm{E}+21) \mathrm{kg}$. The average mass of particles (radiation quanta) is about E-29kg. Radiation frequency is equal to approx. $v=\frac{m c^{2}}{h}=\frac{10^{-29} \cdot 9 \cdot 10^{16}}{6,6 \cdot 10^{-34}} \approx 10^{21} 1 / \mathrm{s}$. This is the bottom boundary of hard gamma radiation [16]. Thus the volume of information in the neutron star of the mass of the
Sun $(\sim 2,38 \mathrm{E}+59$ bit $)$ is sufficient for the formation of a black hole of the mass of $\sim 3 \mathrm{E}+24 \mathrm{~g}$.

Suppose that the original white dwarf possesses the mass equal to the mass of the Sun $M_{\text {Sun }}=2,00 \mathrm{E}+30 \mathrm{~kg}$ and contains the information volume equal to $1,24 \mathrm{E}+59$ bit. Because $\quad n_{e} \log _{2} n_{e}+n_{C+O} \cdot 129,5 \geq \frac{k(k+1)}{2} \approx \frac{k^{2}}{2}$

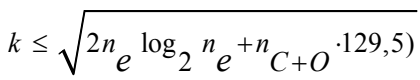

and $M_{B h}=k m_{0} \leq m_{0} \sqrt{\left.2 n_{e} \log _{2} n_{e}+n_{C+O} \cdot 129,5\right)}$

(restriction on the mass of a black hole formed from the white dwarf). When the volume of information in the white dwarf is equal to $1,14 \mathrm{E}+59$ bit. $k \leq 4,8 \cdot 10^{29}$. Because $m_{0}$ $=5,09 \mathrm{E}-09 \mathrm{~kg}$, then $M_{B h} \leq m_{0} 5 \cdot 10^{29} \approx 2,4 \cdot 10^{21} \mathrm{~kg}$.

Suppose that the original star is the star of the Sun type having the mass equal to the mass of the Sun

$M_{n}=2,00 \mathrm{E}+30 \mathrm{~kg}$ and contains the volume of information equal to $\approx 1,3 \cdot 10^{58}$ bit. The mass of the newly formed black hole is equal to $M_{B h}=k m_{0}$, the volume of information in the newly formed black hole is equal to $I=\frac{k(k+1)}{2} \approx \frac{k^{2}}{2}$. Because the star of the Sun type contains only the usual substance, then the mass of the black hole under formation is equal to $M_{B h}=m_{0} k=m_{0} \sqrt{2 I_{U S}}$ In the case under study

$$
\begin{aligned}
& M_{B h}=m_{0} \sqrt{2 I_{U}} \approx 5,09 \cdot 10^{-9} \sqrt{2 \cdot 1,3 \cdot 10^{58}}=5,09 \cdot 10^{-9} \sqrt{2,6 \cdot 10^{58}} \approx \\
& \approx 5,09 \cdot 10^{-9} \cdot 1,6 \cdot 10^{29} \approx 8 \cdot 10^{20} \mathrm{~kg} .
\end{aligned}
$$

One can see from the table 3 that the mases of the black holes that can be generated during formation of black holes from neutron star, white dwarf, star of the Sun type are close to the mass of the optimal black hole (in the system "black hole - radiation") - $8,08 \mathrm{E}+22 \mathrm{~kg}$.

Table 3. Mass of the newly formed black hole

\begin{tabular}{|c|c|c|c|c|}
\hline Star type & Star mass & $\begin{array}{c}\text { Volume of information } \\
\text { in the star }\end{array}$ & $\begin{array}{c}\text { Number of sub Planck particles } \\
\text { in the newly formed black hole }\end{array}$ & $\begin{array}{c}\text { Mass of the newly } \\
\text { formed black hole } \\
(\mathrm{kg})\end{array}$ \\
\hline Neutron star & $\begin{array}{c}1,0 \text { of the mass of the } \\
\text { Sun }\end{array}$ & $2,38 \mathrm{E}+59$ & $7 \mathrm{E}+29$ & $3,5 \mathrm{E}+21$ \\
\hline Neutron star & $\begin{array}{c}1,4 \text { of the mass of the } \\
\text { Sun }\end{array}$ & $3,35 \mathrm{E}+59$ & $8 \mathrm{E}+29$ & $4,17 \mathrm{E}+21$ \\
\hline White dwarf & $\begin{array}{c}1,0 \text { of the mass of the } \\
\text { Sun }\end{array}$ & $1,24 \mathrm{E}+59$ & $4,9 \mathrm{E}+29$ & $2,5 \mathrm{E}+21$ \\
\hline Star of the Sun type & $\begin{array}{c}1,0 \text { of the mass of the } \\
\text { Sun }\end{array}$ & $1,3 \mathrm{E}+58$ & $1,2 \mathrm{E}+29$ & $8 \mathrm{E}+20$ \\
\hline
\end{tabular}




\section{Black Hole Merger}

Let us consider a system consisting of the two black holes. Suppose that the black holes have the mass of $M_{1}=n_{1} m_{0}$, $M_{2}=n_{2} m_{0} \quad\left(m_{0}\right.$ - the minimal black hole mass, $\left.n_{1}, n_{2}=1,2, \ldots\right)$ and contain the volume of information equal to $I_{1}=\frac{n_{1}\left(n_{1}+1\right)}{2}$ and $I_{2}=\frac{n_{2}\left(n_{2}+1\right)}{2}$. The mass of the system is equal to $M_{1+2}=\left(n_{1}+n_{2}\right) m_{0}$, the volume of information in the system is $I_{1+2}=\frac{n_{1}\left(n_{1}+1\right)}{2}+\frac{n_{2}\left(n_{2}+1\right)}{2}$. Is it possible that a black hole is formed as a result of the two given black holes merger and only two given black holes? The mass of a new black hole must be equal to the sum of the masses of the original black holes $M=M_{1+2}=\left(n_{1}+n_{2}\right) m_{0}$. The volume of information in the new black hole must be equal to $I=\frac{\left(n_{1}+n_{2}\right)\left(n_{1}+n_{2}+1\right)}{2}$. It is obvious that under no masses of the original black holes the volume of information in the newly formed black hole would coincide with the total volume of information in the original black holes

$$
\begin{aligned}
& I=\frac{\left(n_{1}+n_{2}\right)\left(n_{1}+n_{2}+1\right)}{2}=\frac{n_{1}^{2}+n_{1} n_{2}+n_{1}+n_{1} n_{2}+n_{2}^{2}+n_{2}}{2} \neq \\
& \neq \frac{n_{1}^{2}+n_{1}+n_{2}^{2}+n_{2}}{2}=\frac{n_{1}\left(n_{1}+1\right)}{2}+\frac{n_{2}\left(n_{2}+1\right)}{2}=I_{1}+I_{2} .
\end{aligned}
$$

Let us consider the following example. Suppose that the mass of the black holes is $M_{1}=M_{2}=m_{0}$ and they contain the volume of information equal to $I_{1}=I_{2}=1$. The mass of the system is $M_{1+2}=2 m_{0}$, the volume of information in the system is $I_{1+2}=1+1=2$.

Is it possible that a black hole is formed as a result of merging the two black holes of the minimal mass and only the two given black holes of the minimal mass? The mass of a new black hole must be equal to the sum of masses of the original black holes $M=M_{1+2}=2 m_{0}$. The volume of information in a new black hole must be equal to $I=\frac{(1+1)(1+1+1)}{2}=3$. Apparently the volume of information in a new black hole does not coincide with the aggregated volume of information in the original black holes.

We give a system of equations for estimation of the mass of a black hole under formation when the two black holes are merged.

Let us suppose that the black holes before merging possess the mass of $M_{1}=n_{1} m_{0}, M_{2}=n_{2} m_{0} \quad\left(m_{0}=5,09 \mathrm{E}-09 \mathrm{~kg}-\right.$ is the minimum mass of the black hole $\left.n_{1}, n_{2}=1,2, \ldots\right)$ and contains the volume of information equal to $I_{1}=\frac{n_{1}\left(n_{1}+1\right)}{2}$ and $I_{2}=\frac{n_{2}\left(n_{2}+1\right)}{2}$. As a result of the original black holes a black hole and usual substance are created. The mass of the black hole after the merger is equal to $M_{1+2}=n m_{0}$, the mass of information in the newly formed black hole is equal to $I_{1+2}=\frac{n(n+1)}{2}$. A number of particles of usual substance that have been formed after the merger is equal to $x$. The volume of information in the usual substance is equal to $I_{U s}=x$. The average mass of a particle of usual substance is equal to $m$.

From the law of conservation of uncertainty (information) it follows:

$$
I_{1}+I_{2}=\frac{n_{1}\left(n_{1}+1\right)}{2}+\frac{n_{2}\left(n_{2}+1\right)}{2}=\frac{n(n+1)}{2}+x=I_{1+2}+I_{U S} .
$$

We believe that the main energy of black holes and usual substance is concentrated in the mass. Then from the energy conservation principle it follows:

$$
M_{1}+M_{2}=\left(n_{1}+n_{2}\right) m_{0}=n m_{0}+x m .
$$

Thus we have a two-equations system

$$
\begin{gathered}
\frac{n_{1}\left(n_{1}+1\right)}{2}+\frac{n_{2}\left(n_{2}+1\right)}{2}=\frac{n(n+1)}{2}+x, \\
\left(n_{1}+n_{2}\right) m_{0}=n m_{0}+x m \text {, with three unknowns } n, x, \\
m . x \geq 0 \text {. Hence, } \\
n>1 \\
n^{2} \leq n_{1}^{2}+n_{2}^{2} \text { (restriction on the mass of the black }
\end{gathered}
$$
hole after merging).

Next we have

$$
\begin{gathered}
n_{1}^{2}+n_{1}+n_{2}^{2}+n_{2}-n^{2}-n=2 x \quad,\left(n_{1}+n_{2}\right) m_{0}=n m_{0}+x m . \\
\text { And } x=\frac{n_{1}^{2}+n_{1}+n_{2}^{2}+n_{2}-n^{2}-n}{2}, \\
m=\frac{\left(n_{1}+n_{2}-n\right) m_{0}}{x}=\frac{2\left(n_{1}+n_{2}-n\right)}{n_{1}^{2}+n_{1}+n_{2}^{2}+n_{2}-n^{2}-n} m_{0} . \\
\text { At } n>>1 \quad m \approx \frac{2 m_{0}}{n} .
\end{gathered}
$$

The following assertions are valid.

Assertion 1. A black hole cannot be created by means of merging the two black holes and only two black holes. Merging of black holes can occur only with the absorption and emission of usual substance.

Assertion 2. A black hole cannot be created by means of 
merging $\mathrm{k}$ black holes and only $\mathrm{k}$ black holes. Merging of black holes can occur only with extra absorption and emission of usual substance.

Assertion 3. A black hole can reduce its mass through emission of usual substance and only it into space. A black hole can increase its mass through absorption of usual substance and only it from space. During the two black holes merger one of them should emit usual substance into space while another one should absorb usual substance from space.

Assertion 4. During the merger of two black holes having the masses $M_{1}=n_{1} m_{0}, M_{2}=n_{2} m_{0}$, without using any additional usual substance, the mass of a newly formed black hole is less than $M_{1+2}=\sqrt{n_{1}\left(n_{1}+1\right)+n_{2}\left(n_{2}+1\right)} m_{0} \approx \sqrt{n_{1}^{2}+n_{2}^{2}} m_{0}=\sqrt{M_{1}^{2}+M_{2}^{2}}$

During the merger of two black holes having the same masses $M_{1}=M_{2}=n m_{0}$, the mass of a newly formed black hole is less than

$$
M_{1+2}=\sqrt{2 n^{2}} m_{0}=n m_{0} \sqrt{2}=\sqrt{2} M_{1}=\sqrt{2} M_{2} \text {. The mass }
$$
of a black hole that was formed as a result of the merger of 2 black holes of the same masses without using any additional usual substances is $\sqrt{2}$ times less than the sum of masses of merging black holes. The remaining mass is dissipated in the space.

Assertion 5. During the merger of $k$ black holes having the mass of $M_{i}=n_{i} m_{0}$ without using any additional usual substance, the mass of a newly formed black hole is less than $M_{1+2+\ldots+k}=m_{0} \sqrt{\sum_{i=1}^{k} n_{i}\left(n_{i}+1\right)} \approx m_{0} \sqrt{\sum_{i=1}^{k} n_{i}^{2}}=\sqrt{\sum_{i=1}^{k} M_{i}^{2}}$

During the merger of $k$ black holes of the same masses $M_{i}=n m_{0}=M \quad$ without using any additional usual substance, the mass of a newly formed black hole is les than

$$
M_{1+2+\ldots+k}=\sqrt{k n^{2}} m_{0}=n m_{0} \sqrt{k}=\sqrt{k} M \text {. The mass of a }
$$

black hole that was formed as a result of the merger of $\mathrm{k}$ black holes having the same masses is $\sqrt{k}$ times less than the sum of masses of merging black holes. The remaining mass is dissipated in the space.

A system consisting of the two black holes and usual substance. Let us consider a system consisting of the two black holes and usual substance. The black holes possess the same mass $M_{1,0}=n m_{0}, M_{2,0}=n m_{0}$ and contain the same volume of information $I_{1,0}=\frac{n(n+1)}{2}$ and $I_{2,0}=\frac{n(n+1)}{2}$. The usual substance is represented by the photons having different frequencies (energy) $E=h v$, each of them according to the foundational principle of Zeilinger quantum mechanics contains one bit of information.

Assertion 6. During the merger of $k$ black holes having the same masses $M_{1}=n m_{0}$ and $M_{2}=n m_{0} \quad$ and containing the same volumes of information $I_{1}=\frac{n(n+1)}{2}$ and $I_{2}=\frac{n(n+1)}{2}$, the absorbing black hole must absorb by $n^{2}$ bit information more than contained in the absorbed black hole $I_{2}=\frac{n(n+1)}{2}$ - the absorbing black hole must absorb additionally $n^{2}$ particles of usual substance, each of them containing one bit of information.

Assertion 7. The merger of the two black holes having the masses of $M_{1}=n_{1} m_{0}$ and $M_{2}=n_{2} m_{0}$ and containing the volumes of information $I_{1}=\frac{n_{1}\left(n_{1}+1\right)}{2} \quad$ and $I_{2}=\frac{n_{2}\left(n_{2}+1\right)}{2}$ resulting in one black hole formation, requires the usage of extra $n_{f}=n_{1} n_{2}$ radiation quanta $n_{1} n_{2}$ particles of usual substance each of them containing one bit of information.

\section{Estimation of the Volume of Information in the Planck Particle}

Energy necesary for formation of one bit is not less than $k T \ln 2[6,17]$. Energy necessary for formation of one Nat is not less than $k T$.

Mass necessary for formation of one bit is not les than $\frac{k T \ln 2}{c^{2}}$. Mass necessary for formation of one Nat is not less than $\frac{k T}{c^{2}}$. At Planck temperature $T_{P l}=\frac{1}{k} \sqrt{\frac{\hbar c^{5}}{G}}=1,41696 \cdot 10^{32} \mathrm{~K}[18]$ the mass necessary for formation of one Nat is not less than Planck mass $m=\frac{k T}{c^{2}}=\sqrt{\frac{\hbar c}{G}}=m_{P l}=2,17671 \cdot 10^{-5} \mathrm{~g}$, the mass necessary for formation of one bit is not less than $m_{P l} \ln 2 \approx 0,69 m_{P l}=1,5 \cdot 10^{-5} \mathrm{~g}$.

«Fourteen billion years ago at birth of the Universe it was enclosed in the point with the radius of $10^{-33} \mathrm{~cm}$, which is incommensurably smaller then the proton radius $-10^{-13} \mathrm{~cm}$. In that volume all the information about the future of the Universe had already been built-in. The Big Bang occurred» (A.Cherepashuk [19]). $10^{-33} \mathrm{~cm}-$ is the size of a Planck particle. The Planck particle contains one Nat of 
information $(\approx 1,45$ bit $)$ while the information about the Universe contains not less than $\approx 10^{14}$ bit of classical information. Hence, all the information about the future of the Universe was enclosed in the Universe segment of the radius larger than $10^{-33} \mathrm{~cm}$. It is not impossible that similar informational considerations would allow to prove either independent development of the Universe determined by the information (heterogeneity) contained in it, or the presence of additional external control executed from outside of the Universe.

\section{Conclusion}

The results presented in this paper show the effectiveness of informational approach for studying the cosmological objects.

1. We consider the following system of q-bits: non interacting q-bits in the system, the system having $\mathrm{n}$ basic states, q-bits pairwise interaction in the system, local q-bit interaction in the system.

2. It is shown that in the general case, the volume of information $I_{n}$ in the system of $n$ q-bits is larger or equal to zero bits and is not larger than $\mathrm{n}(\mathrm{n}+1) / 2$ bit.

3. It is shown that the information volume contained in the black hole is proportional to its squared mass. A black hole is the aggregate of particles (black particles) each having a mass equal to 0.23 th of Planck mass and interacting with all other black particles that form a black hole. The estimates of black holes distribution by mass one can calculate the intensity of aggregated distribution of black holes radiation by frequencies and compare them with the experiment results. From the obtained radiation frequency expression one can draw the estimate of black hole radiation temperature. Are given information characteristics of black holes of different masses.

4. The neutron star with the mass of the Sun contains $\approx 2,32 \cdot 10^{59}$ bit.

5. The white dwarf with the mass of the Sun contains $\approx 1,24 \cdot 10^{59}$ bit.

Estimate of dependence of the temperature of neutron star radiation on its mass (number of neutrons in the star).

6 . The Sun contains $\approx 1,3 \cdot 10^{58}$ bit of information.

7. The mases of the black holes that can be generated during formation of black holes from neutron star, white dwarf, star of the Sun type are close to the mass of the optimal black hole (in the system "black hole - radiation").

8. A black hole cannot be created by means of merging some black holes and only some black holes. Merging of black holes can occur only with the absorption and emission of usual substance. The mass of a black hole that was formed as a result of the merger of 2 black holes of the same masses without using any additional usual substances is $\sqrt{2}$ times less than the sum of masses of merging black holes. The remaining mass is dissipated in the space.

9. All the information about the future of the Universe was enclosed in the Universe segment of the radius larger than $10^{-33} \mathrm{~cm}$.

10. In future the proposed models and estimations should undoubtedly be specified and presented in more detailed way.

\section{REFERENCES}

[1] Zeilinger A. A Foundational Principle for Quantum Mechanics", Foundations of Physics 29 (4): 631-43. (1999),

[2] Shannon C. A Mathematical Theory of Communication // Bell System Technical Journal. (1948) T. 27. p. 379-423, 623-656.

[3] Stratonovich R.L. Information Theory. (In Russian). Moscow: Soviet Radio, 1975.

[4] Gurevich I.M. Law of informatics - a basis of researches and designing of complex communication and management systems. (In Russian) «Ecos». Moscow (1989) 60 p.

[5] Gurevich I.M. «Informatics Laws - a basis of a structure and cognitive of complex systems». (In Russian) The second edition. Moscow. «Torus Press». (2007).

[6] Valiev K.A. Kokin A.A. Quantum computers: Hope and reality. (In Russian) Scientific and publishing center "Regular and chaotic dynamics". Moscow-Izhevsk (2004) p. 320 .

[7] Shapiro S., Teukolsky S. Black Holes, White Dwarfs and neutron Stars. Cornell University, Ithasa, New York. (1983).

[8] Gurevich I.M. Information characteristics of the entanglement states. (In Russian). Magazine Information technologies. № 5. Moscow. 2006.

[9] Penrose R. The Emperor's New Mind. Oxford University Press. (1989) 466 p.

[10] Gurevich I.M. Information model of a black hole. (In Russian). Works of the conference BAK-2007. 2007. Kazan.

[11] June gamma-ray burst did not fit into the theory. http://elementy.ru/news/430418.

[12] Gurevich I.M. On information models in cosmology. (In Russian). Systems and Means of Informatics. Issue 17. IPI RAN. Moscow. 2007. pp. 164-183.

[13] Gurevich I. About restrictions on volume of the information in the Universe. 58 th International Astronautical Congress 2007 (IAC 2007).

[14] Lloyd S. Computational capacity of the Universe. arXiv:quant-ph/0110141 v1 24 Oct 2001.

[15] http://crydee.sai.msu.ru/ mir/Star_Life.site/Structure/Star_m odels/Dwarfs/wdmod.htm

[16] http://elementy.ru/posters/spectrum/gamma.

[17] Brillouin L. Science and information theory. (In Russian) Fizmatgiz. Moscow (1960), 392 p.

[18] Tomilin K. A. Plank's quantities. 100 years of quantum 
theory. History. Physics. Philosophy. (In Russian) Works of the international conference, NIA-Nature. Moscow. 2002. pp.105-113.
[19] Leskov S. It's find a place for the God. (In Russian). 2006. http://izvestia.ru/news/310380 